\title{
Role of SIRT2 in Regulation of Stemness of Cancer Stem-Like Cells in Renal Cell Carcinoma
}

\author{
Ruojing Wei Dalin He Xinshi Zhang \\ Department of Urological Surgery, the First Affiliated Hospital of Xian Jiaotong University, Xi,an, China
}

\section{Key Words}

Cancer stem cells $(\mathrm{CSCS}) \cdot \mathrm{SIRT} 2 \cdot$ Renal cell carcinoma (RCC)

\begin{abstract}
Background/Aims: Cancer stem cells (CSCs) contribute to tumorgenesis, invasion and metastasis, and are typically resistant to chemotherapy. Recent reports showed that SIRT2 was upregulated in several cancers. However, whether SIRT2 may be a CSC marker in renal cell carcinoma (RCC) is not clear. Methods: The SIRT2 levels in both RCC samples and the corresponding normal kidney samples (NT) were assessed by RT-qPCR and ELISA. The association between SIRT2 levels and patient survival was examined using Bivariate correlation analysis by Spearman's Rank Correlation Coefficients. The survival of the patients was analyzed using Kaplan-Meier curve. In vitro, 2 RCC cell lines were co-transduced with a lentivirus expressing both a green fluorescent protein and a luciferase reporter under a cytomegalovirus promoter, and another lentivirus expressing a nuclear red fluorescent protein reporter under the control of a SIRT2 promoter for differentiating SIRT2+ vs SIRT2- RCC cells by flow cytometry. The SIRT2+ vs SIRT2- RCC cells were examined for the potential of forming tumor sphere in a tumor sphere formation assay, resistance to fluorouracil-induced apoptosis by CCK-8 assay, and the frequency of forming tumor in vivo after serial adoptive transplantation by bioluminescence. Results: The levels of SIRT2 were higher in RCC samples than NT. The prognosis of RCC patients with high SIRT2 was worse than that of with low SIRT2. Compared to SIRT2- cells, SIRT2+ cells formed more tumor spheres, appeared to be more resistant towards fluorouracil-induced apoptosis, and generated bigger tumors with higher frequency after serial adoptive transplantation. Conclusion: SIRT2 may be highly expressed in the RCC stem-like cells and regulates cancer metastasis. Selective knockout of SIRT2 or elimination of SIRT2+ cells may improve the therapeutic outcome for patients with RCC.
\end{abstract}




\section{Cellular Physiology Cell Physiol Biochem 2018;49:2348-2357 \begin{tabular}{ll|l} 
and Biochemistry Published onlIne: 28 September 2018 & $\begin{array}{l}\text { (c) } 2018 \text { The Author(s). Published by S. Karger AG, Basel } \\
\text { www.karger.com/cpb }\end{array}$ \\
\hline
\end{tabular} \\ Wei et al.: SIRT2 as a CSC Marker in RCC}

\section{Introduction}

Renal cell carcinoma (RCC) is a common type of human cancer [1-3]. RCCs are typically found to grow within a kidney, while more than 2 tumors in one kidney or even tumors in both kidneys have been detected in previous reports [1-3]. The outcome of RCC therapy largely relies on the presence of metastases of the primary tumor or not, thus the molecular mechanisms underlying the migration and invasiveness of RCC appear to be the top question in the study of RCCs.

Cancerous cells metastasis has resulted in the distal tumor by migration [4]. The specific cancer stem cells (CSCs) were reported to support the development, metastasis and chemoresistance in RCC [5]. Currently, standard first-line chemotherapy for metastatic RCC was fluorouracil (5-FU) [6]. The CSCs was identified with surface and non-surface biomarkers, which were conserved and specific. Specifically, the identical biomarkers can be observed in several different cancers, while the specific biomarkers can also be detected in some cancers [7]. For RCC, prominin-1 (CD133) and CD24 were the common biomarkers for CSCs [8-10]. Further, the "CSCs" in this study was collected in RCC based on the most common biomarkers, which was only enriched CSC population. Thus, these CSCs could only be regarded as CSClike cells. Further purification should be performed with more RCC specific biomarkers for obtaining more pure CSCs.

The yeast silent information regulator 2 (SIR2) is a histone deacetylase that modifies functions of nicotinamide adenine dinucleotide [11-13]. In mammals, there are 7 homologs of SIR2 (SIRT1-7) [11-13]. Recently, SIRT2 was found to be associated with mitotic cell cycle control [11-13], in which SIRT2 likely promoted tumorigenesis [14-17]. Upregulation of SIRT2 has been detected in a number of tumors [16-22]. However, it is not known whether SIRT2 is a CSC marker in cancers, including RCC. We explored the issue in this study.

Here, The SIRT2 levels in both RCC samples and the corresponding normal kidney samples (NT) were assessed. The association between SIRT2 levels and patient survival was also examined. In vitro, 2 RCC cell lines were co-transduced with a lenti-vector expressing both a green fluorescent protein and a luciferase reporter under a cytomegalovirus promoter, and a lenti-vector expressing a nuclear red fluorescent protein reporter under the control of a SIRT2 promoter for differentiating SIRT2+ vs SIRT2- RCC cells. The SIRT2+ vs SIRT2- RCC cells were examined for the formation of tumor sphere, resistance to fluorouracil-induced apoptosis, and frequency of tumor formation after serial adoptive transplantation. Our results showed that SIRT2 may be highly expressed in the RCC stem-like cells and regulates cancer metastasis.

\section{Materials and Methods}

\section{Objective}

The RCC samples and corresponding normal tissues of 54 patients were collected in our hospital from 2006 through 2011. The written informed consent was obtained from all the patients to inform the research purpose of this study. The design of this study has been reviewed and approved by the institutional review board of the First Affiliated Hospital of Xian Jiaotong University.

Cell culture

From American Type Culture Collection (ATCC, Rockville, MD, USA), human RCC cell line A-498 (ATCC HTB-44; simplified as HTB) and Hs 891.T (ATCC CRL-7629; simplified as CRL) were purchased. These cells were maintained in DMEM (Invitrogen, USA) suppled with $10 \%$ fetal bovine serum (FBS; Sigma-Aldrich, Rockville, MD, USA). The cells were cultured in an incubator under $37^{\circ} \mathrm{C}$ and $5 \% \mathrm{CO}_{2}$. 5-FU (Sigma-Aldrich) was prepared in the concentration of $1 \mathrm{mmol} / \mathrm{l}$ and applied in the concentration of $2 \mu \mathrm{mol} / \mathrm{l}[23,24]$. Analysis was done 24 after 5-FU treatment. 


\section{Cellular Physiology Cell Physiol Biochem 2018;49:2348-2357

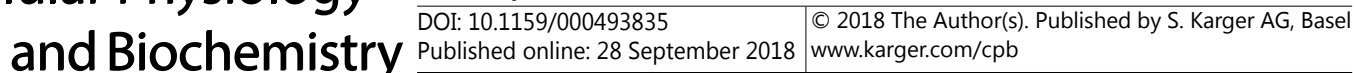

Wei et al.: SIRT2 as a CSC Marker in RCC

\section{Cell transfection and detection}

The RCC cell lines were co-transfected with 2 lentiviruses. The first lentivirus carries a luciferase (LUC) and a green fluorescent protein (GFP) reporter with a promotor from cytomegalovirus (CMV). The LUC and GFP reporters are connected by an internal ribosome entry site (IRES) sequence to allow co-expression of 2 genes by one promoter. The second lentivirus carries a nuclear red fluorescent protein (nRFP) reporter with a control of SIRT2 promoter. For generating plasmids carrying 2 viruses, a pcDNA3.1-CAG-GFP plasmid, a pcDNA3.1-CAG-nRFP plasmid, and a pcDNA3.1-CMV-luciferase plasmid were used as backbones (Clontech, USA). SIRT2 promoter was amplified with PCR with the template of human genomic DNA. For producing the lentivirus, $5 \mu \mathrm{g}$ of prepared plasmids and $5 \mu \mathrm{g}$ each of packaging plasmids (REV, pMDL and VSV-G) were cotransfected into seeded HEK293T cells (ATCC) with Lipofectamine-3000 assay. After transfecting, the cells were incubated for $48 \mathrm{~h}$. Then the supernatant was collected and filtered with the membrane pore size of $0.45 \mu \mathrm{m}$. The obtained filter of lentivirus was further isolated and titrated.

The RCC cell lines were transfected in vitro for viral infection with the multiplicity of infection (MOI) of 80. After incubating for $48 \mathrm{~h}$, the viral infection was stopped. Transduced cells were separated and detected based on the expression of GFP/nRFP and luciferase activity in vitro.

\section{Animal manipulation}

The nude mice were applied to study the tumor growth of treated RCC cells. Male nude mice at an age of 12 weeks were include in this study (SLAC Laboratory Animal Co. Ltd, Shanghai, China). Tumor cells were implanted and serial adoptive transferred subcutaneously for 3 rounds with 20 cells isolated from the previous round. The tumor formation was monitored and quantified with bioluminescence of animals for 8 weeks after transplantation. The bioluminescence detection system was applied to quantifying tumor mass in living animals (IVIS imaging system, USA). The animal was injected with intraperitoneal for $10 \mathrm{~min}$ at 150 $\mathrm{mg} / \mathrm{kg}$ body weight. The image was taken with an acquisition time of 1 minute and a binning value of 10 . During image acquisition, mice were sedated continuously via inhalation of $3 \%$ isoflurane. Image analysis and bioluminescent quantification was performed using Living Image software (Xenogen Corp).

\section{The culture of primary tumor sphere}

The purified RCC cells were removed in tumor sphere media (R\&D Systems, Shanghai, China), for which the cells were cultured in ultra-low attachment 24-well plates (Corning, NY, USA) with a density of $2 \mathrm{X} 10^{4}$ cells per well. The tumor sphere media are consisting of a serum-free DMEM, human recombinant EGF $(20 \mathrm{ng} / \mathrm{ml}), \mathrm{bFGF}(20 \mathrm{ng} / \mathrm{ml})$, leukemia inhibitory factor $(10 \mathrm{ng} / \mathrm{ml})$ and $\mathrm{N}$-acetylcysteine $(60 \mu \mathrm{g} / \mathrm{ml})$. The tumor sphere was observed and analyzed after 1 week.

\section{Cell viability assay}

The cell viability was detected with Cell Counting Kit-8 (CCK-8) assay according to the manufacture's instruction (Sigma-Aldrich). The assay is a sensitive colorimetric assay for determining cell viability in cell proliferation and cytotoxicity assays. Dojindo's highly water-soluble tetrazolium salt, WST-8, is reduced by dehydrogenase activities in cells to give a yellow-color formazan dye, which is soluble in the tissue culture media. The amount of the formazan dye, generated by the activities of dehydrogenases in cells, is directly proportional to the number of living cells. The absorbance value at $450 \mathrm{~nm}$ was recorded with microplate reader. The cell viability was expressed as: the percentage of absorbance value of samples to that of control.

\section{TUNEL assay}

The RCC cells were sorted and analyzed with flow cytometry. The terminal deoxynucleotidyl transferase (TdT)-mediated dUTP nick-end labeling (TUNEL) was performed using a TUNEL Assay kit, as instructed by the manufacturer (R\&D Systems). TUNEL assay has been designed to detect apoptotic cells that undergo extensive DNA degradation during the late stages of apoptosis. The method is based on the ability of TdT to label blunt ends of double-stranded DNA breaks independent of a template. The positive controls used 10 minutes' incubation at room temperature with $1500 \mathrm{U} / \mathrm{ml}$ DNAse 1 in $50 \mathrm{mM}$ Tris pH 7.5, $10 \mathrm{mM} \mathrm{MgCl} 2$ and $1 \mathrm{mg} / \mathrm{ml} \mathrm{BSA}$. 
RT- $q P C R$

The RT-qPCR was applied to quantify the expression levels of SIRT2 with the commercial SYBR Green PCR Kit (Qiagen, Shanghai, China) and the designed primers. The primers for quantifying SIRT2 are 5'-CCGGCCTCTATGACAACCTA-3' and 5'-GGAGTAGCCCCTTGTCCTTC-3'. The primers for quantifying $\beta$-actin are 5'-CTCTTCCAGCCTTC CTTCCT-3' and 5'-AGCACTGTGTTGGCGTACAG-3'. The levels of gene expression were quantified with the $2^{-\Delta \Delta c t}$ method and normalized with the expression level of $\beta$-actin and controls.

\section{ELISA}

The clinical samples were homogenized and centrifuged for obtaining the supernatant. The total protein was extracted from the supernatant with RIPA buffer (Sigma-Aldrich) and quantified with BCA protein assay (Sigma-Aldrich). ELISA was performed using an anti-human SIRT2 ELISA kit (Abcam 227895, Carlsbad, CA, USA) as instructed.

\section{Statistical analysis}

The data was statistically analyzed with GraphPad Prism 6. The Student's T test was performed to compare the data of 2 groups. The values were expressed as mean \pm standard deviation (SD). When $p<0.05$, the data was considered as significant. Bivariate correlations were calculated by Spearman's Rank Correlation Coefficients. Kaplan-Meier curve was applied to record the survival of RCC patients included in this study.

Fig. 1. High SIRT2 levels are associated with poor RCC patient survival. (AD) SIRT2 levels in 54 clinical samples and corresponding control normal tissues from RCC patients (all at Stage III), which were determined by RT-qPCR (Summary, A; individual values, B) and by ELISA (Summary, individual values, D). (E) The 54 patients were followed-up for 5 years. The patients were classified into high-SIRT2 group $(\mathrm{n}=27)$ and lowSIRT2 group $(\mathrm{n}=27)$, based on the median value. Kaplan-Meier curves were plotted. “*” indicated $\mathrm{p}<0.05$. $\mathrm{N}=54$.

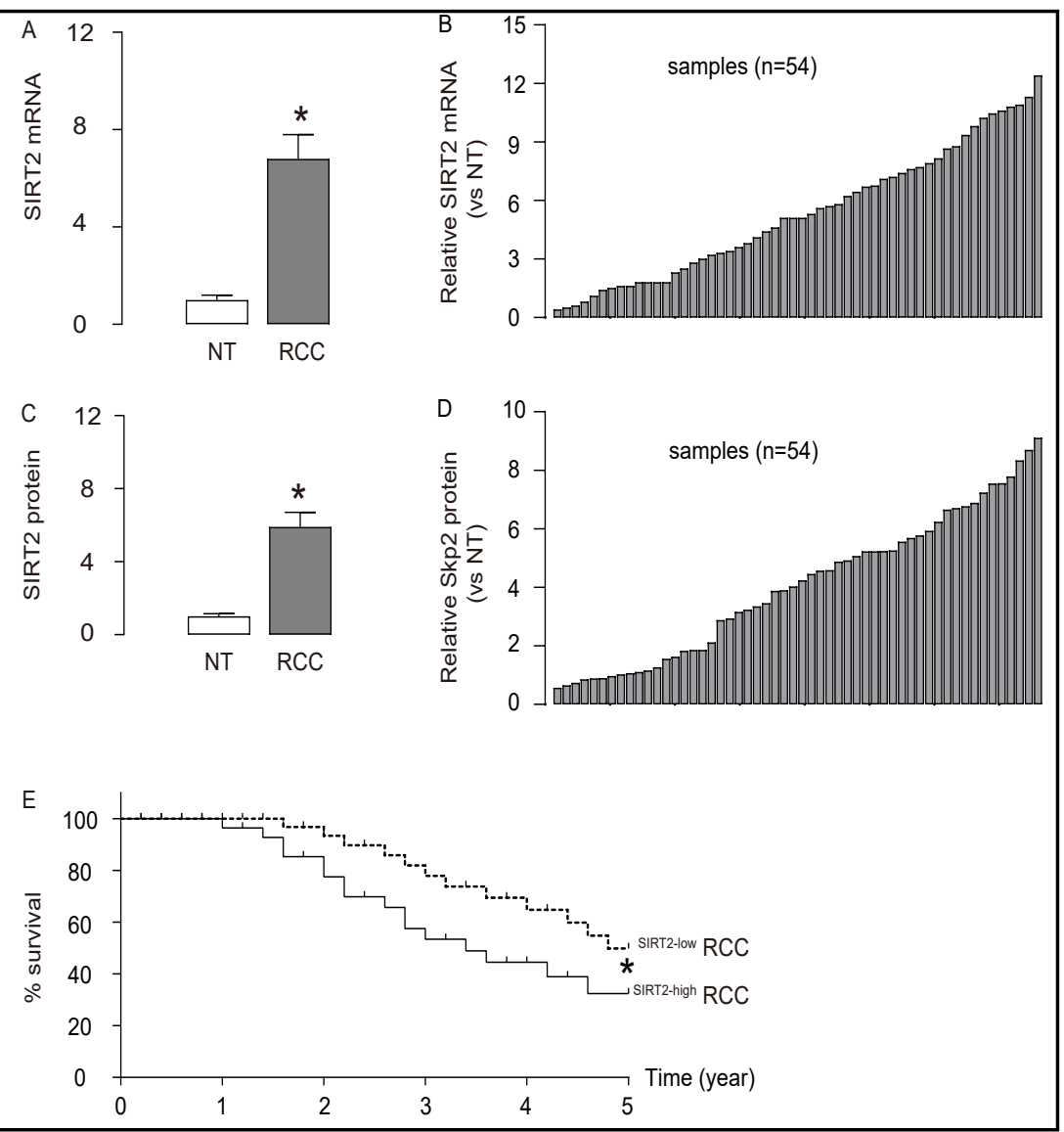




\section{Results}

\section{High SIRT2 levels are associated with poor RCC patient survival}

RCC samples and corresponding normal tissues (NT; $n=54)$ were collected from RCC patients in our hospital (all at Stage III). The levels of SIRT2 levels were determined and compared. Higher expression levels of SIRT2 were detected in RCC samples than NT, by RTPCR (Fig. 1A-B) and by Western blotting (Fig. 1C-D). In addition, the 54 patients were followed up for 5 years to explore the association between the SIRT2 levels in the RCC samples and the overall survival of the patients. The patients were classified into high-SIRT2 group $(n=27)$ and low-SIRT2 group ( $\mathrm{n}=27)$, based on the median value. The overall survival was analyzed with the Kaplan-Meier curves. The results indicated a significantly worse survival for those RCC patients with higher levels of SIRT2 (Fig. 1E).

\section{Genetic labeling of SIRT2+ vs SIRT2-RCC cells with luciferase and different fluorescence}

Two human RCC cell lines (HTB and CRL) were used in culture. These 2 RCC cell lines were co-transduced with 2 lentiviruses. The first lentivirus carries a luciferase (LUC) reporter and a GFP reporter under a CMV promoter (pCMV-LUC-GFP), to label all infected cells with green fluorescence to allow sorting of labelled cells by flow cytometry, and with luciferase to allow the labelled cells to be traced in living animals. The second lentivirus carries a nuclear RFP reporter under the control of a SIRT2 promoter (pSIRT2-nRFP), to allow SIRT2+ and SIRT2- cells to be distinguished and separate by red fluorescence (Fig. 2A). SIRT2 promoter in the virus drives mRFP, while mRFP expression in an infected cell suggests activation of endogenous SIRT2 promoter activity that drives SIRT2 expression in this cell. Thus, expression of mRFP is always detected in cells that express SIRT2. Using a

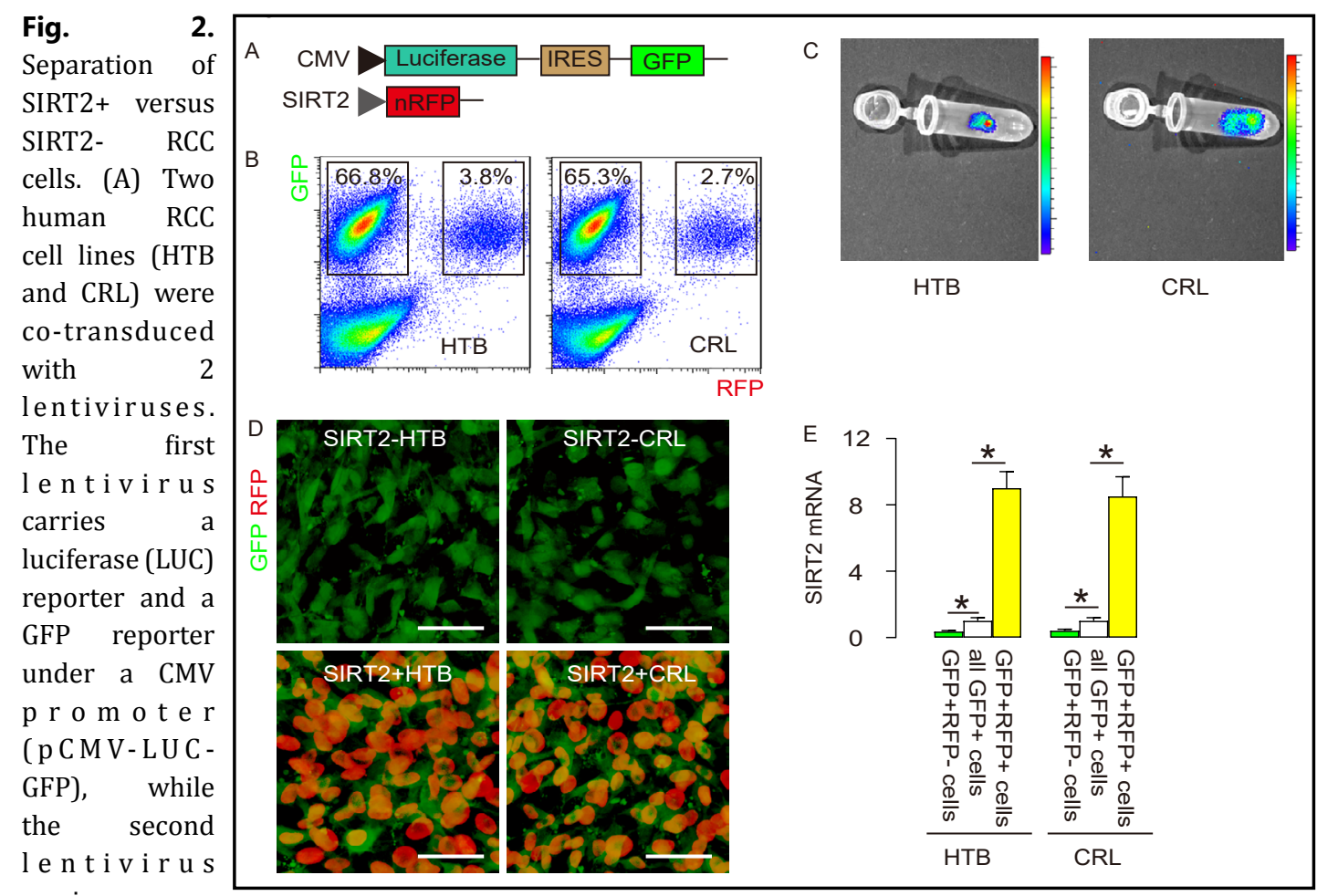
carries a nuclear RFP reporter under the control of a SIRT2 promoter (pSIRT2-nRFP). (B) The transfected RCC cells were separated into different fractions (GFP+RFP+ cells that represent SIRT2+ cells and GFP+RFP- cells that represent SIRT2- cells). (C) The transfected cells were detectable after being treated with luciferin. (D) The transfected cells were purified and examined for fluorescence. (E) RT-qPCR for SIRT2 in different cell fractions. "*" indicated $\mathrm{p}<0.05$. $\mathrm{N}=5$. Scale bars were $20 \mu \mathrm{m}$. 
MOI of 80, both HTB and CRL were transduced with the 2 lentiviruses. The flow cytometry analysis showed that more than half of the RCC cells were transduced and appeared to be GFP+. Among all GFP+ cells, there were a few cells appeared to be RFP+ and thus were yellow fluorescent (positive for both RFP and GFP), and these cells (GFP+RFP+) were SIRT2+ RCC cells. On the other hand, the GFP+RFP- cells (green fluorescent due to absence of RFP) were SIRT2- RCC cells (Fig. 2B). All the transduced cells carried luciferase to be detectable in a luciferin assay in vitro (Fig. 2C). The fluorescent cells (GFP+RFP+ and GFP+RFP-) were then sorted into culture, which confirmed the flow cytometry-based cell sorting (Fig. 2D). To confirm that RFP+ cells were indeed enriched for SIRT2-expressing cells, we examined the mRNA level of SIRT2 in GFP+RFP+ and GFP+RFP- cells, compared to total GFP+ cells. Our data showed that the expression level of SIRT2 mRNA in GFP+RFP+ cells was increased by

Fig. 3. The in vitro CSC characters of SIRT2+ RCC cells. (A-B) The formation of tumor sphere by SIRT2and SIRT2+ cells from two cell lines. (C-E) fluorouracil (5-FU) was applied to treat SIRT2- and SIRT2+ cells from both cell lines. (C) Cell viability with CCK-8 assay. (DE) Cell apoptosis with TUNEL assay, shown by quantification (D), and by representative flow charts (E). (F) Since CD133 is the most well-established CSC marker in renal cancer, we examined CD133 positivity in SIRT2+ and SIRT2fractions by flow cytometry. “*” indicated $\mathrm{p}<0.05$. $\mathrm{N}=5$. Scale bars were $100 \mu \mathrm{m}$.

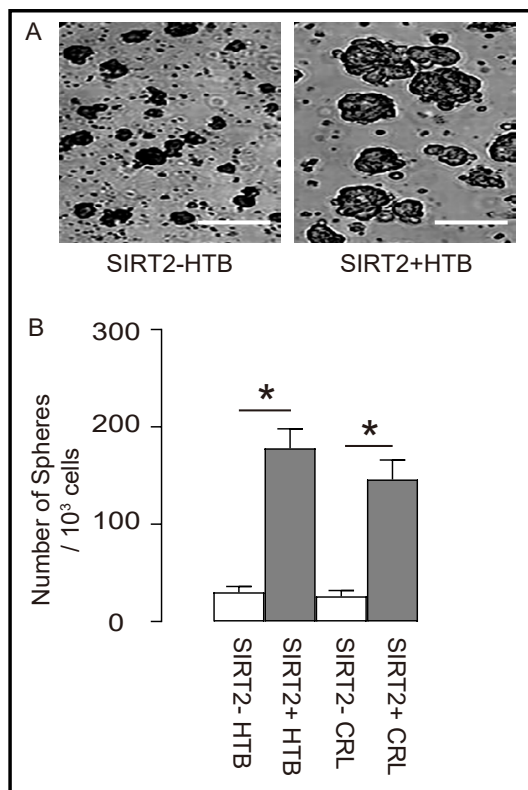

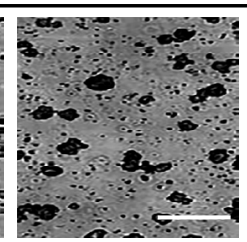

SIRT2-CRL
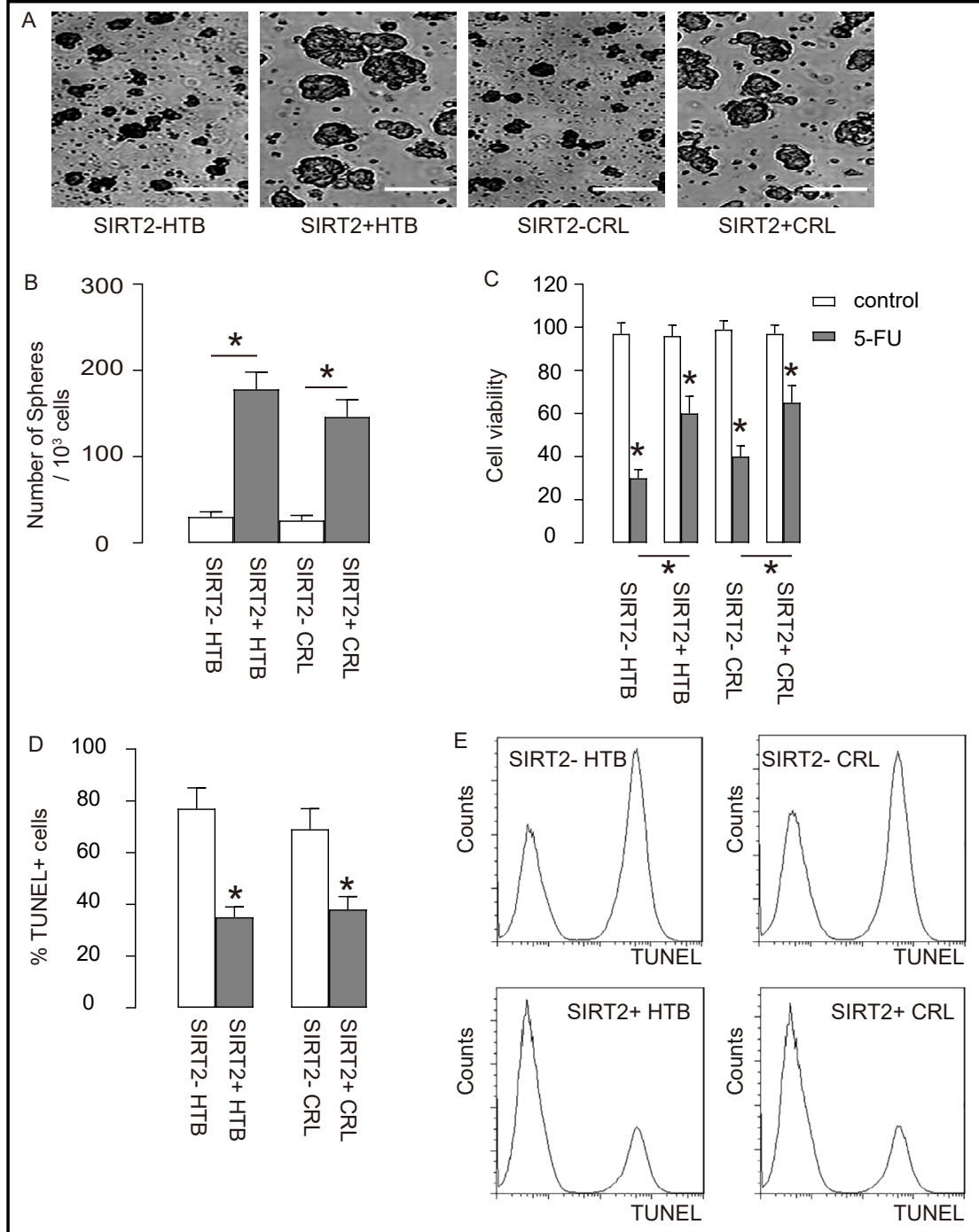
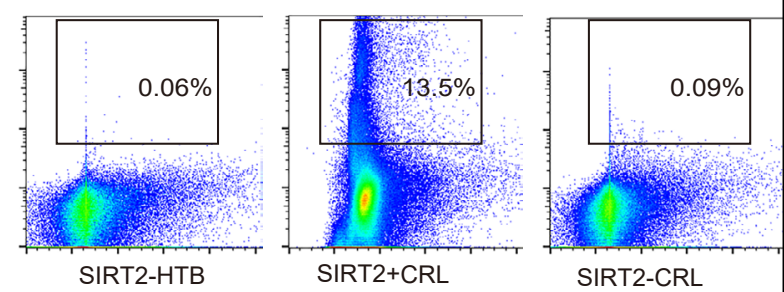


\section{Cellular Physiology Cell Physiol Biochem 2018;49:2348-2357 \\ \begin{tabular}{ll|l} 
and Biochemistry Publisned onIIne: 28 September 2018 & $\begin{array}{l}\text { (c) } 2018 \text { The Author(s). Published by S. Karger AG, Basel } \\
\text { www.karger.com/cpb }\end{array}$ \\
\hline
\end{tabular} \\ Wei et al.: SIRT2 as a CSC Marker in RCC}

more than 8 folds, compared to total GFP+ cells in both lines, while the expression level of SIRT2 mRNA in GFP+RFP- cells was decreased by more than 70\%, compared to total GFP+ cells in both lines (Fig. 2E). These data suggest that RFP+ cells were indeed enriched for SIRT2-expressing cells.

The in vitro CSC characters of SIRT2+ RCC cells

The in vitro CSC characters were examined with two strategies and the SIRT2- cells were applied as controls. Firstly, formation of tumor sphere was tested in both SIRT2- and SIRT2+ cells from both cell lines. It observed that there were significantly more tumor spheres formed in SIRT2+ cells, compared to that of in SIRT2- cells. Similar results were obtained in both cell lines (Fig. 3A and 3B). Secondly, fluorouracil (5-FU) was applied to SIRT2- and SIRT2+ cells from both cell lines and the cell survival was analyzed with CCK-8 assay. 5-FU has been the first line drug of chemotherapy for RCC. Compared to SIRT2- cells, the cell survival with 5-FU was significantly better in SIRT2+ cells (Fig. 3C). The cell apoptosis was reduced through the results of TUNEL assay (Fig. 3D and 3E). Since CD133 is the most well-established CSC marker in renal cancer, finally we examined CD133 positivity in SIRT2+ and SIRT2- fractions. More than $10 \%$ of CD133+ cells were detected in SIRT2+ fraction in both lines, while less than $0.1 \%$ CD133+ cells were detected in SIRT2- fraction in both lines (Fig. 3F). Together, these data suggest that SIRT2+ RCC cells presented with in vitro CSC characters.

Larger tumors were formed in transplanted SIRT2+ RCC cells than SIRT2-RCC cells

The SIRT2- and SIRT2+ cells were transplanted into nude mice for assessment of the tumor formation. Same number of cells was subcutaneously transplanted. The formation of tumor was monitored through the quantification of bioluminescence 8 weeks after tumor implantation. In the monitoring results, significantly larger tumors were observed in SIRT2+ cells-transplanted mice, compared to that of in SIRT2- cells-transplanted mice (Fig. 4A and 4B).

More frequent tumor formation by SIRT2+ RCC cells than SIRT2- RCC cells

The metastasis was characterized with serial adoptive translation in SIRT2+ and SIRT2cells. After the tumor cells were subcutaneously transplanted in the mouse, the GFP+ tumor cells were detected in mouse blood 8 weeks later with flow cytometry. In this study, when there were more than $5 \mathrm{GFP}+$ cells detected in the $10^{6}$ blood cells (with deprived red blood cells), the case was positive. Otherwise, the case was considered negative. In the results, GFP+ cells were more frequently observed in the blood cells of mice transplanted with SIRT2+ RCC cells (Fig. 5A). Then, 20 cells were purified from the primary tumor developed from

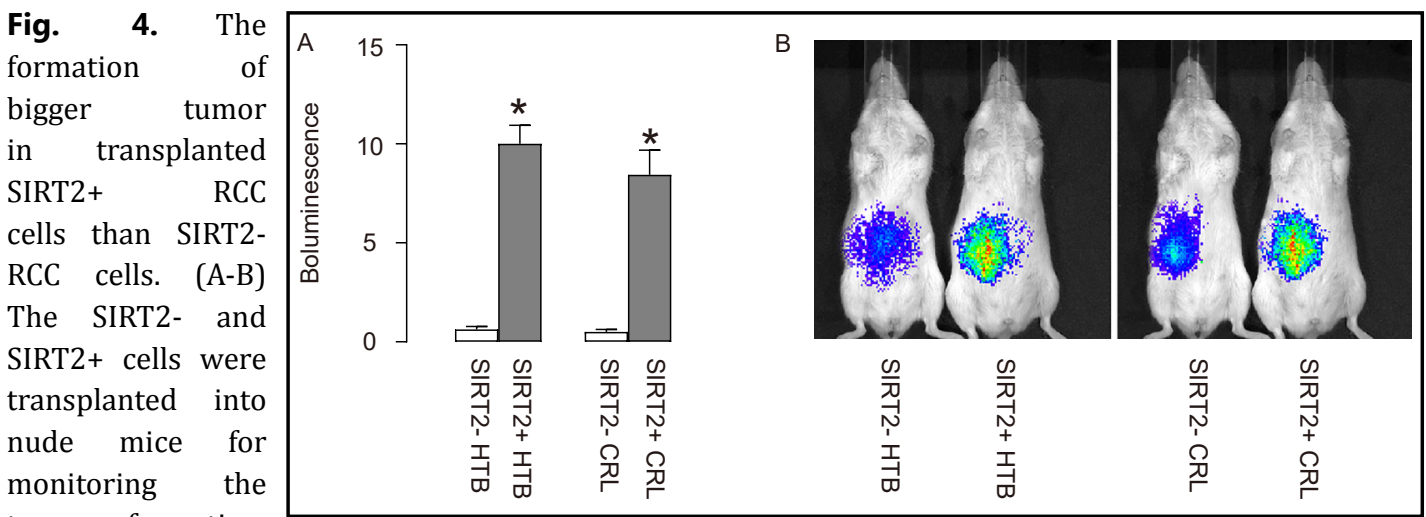

tumor formation.

The formation of

tumor was monitored through the quantification of bioluminescence 8 weeks after tumor implantation. Tumor size was analyzed with bioluminescence, shown by quantification (A), and by representative images (B). “*” indicated $\mathrm{p}<0.05 . \mathrm{N}=5$.

\section{KARGER}




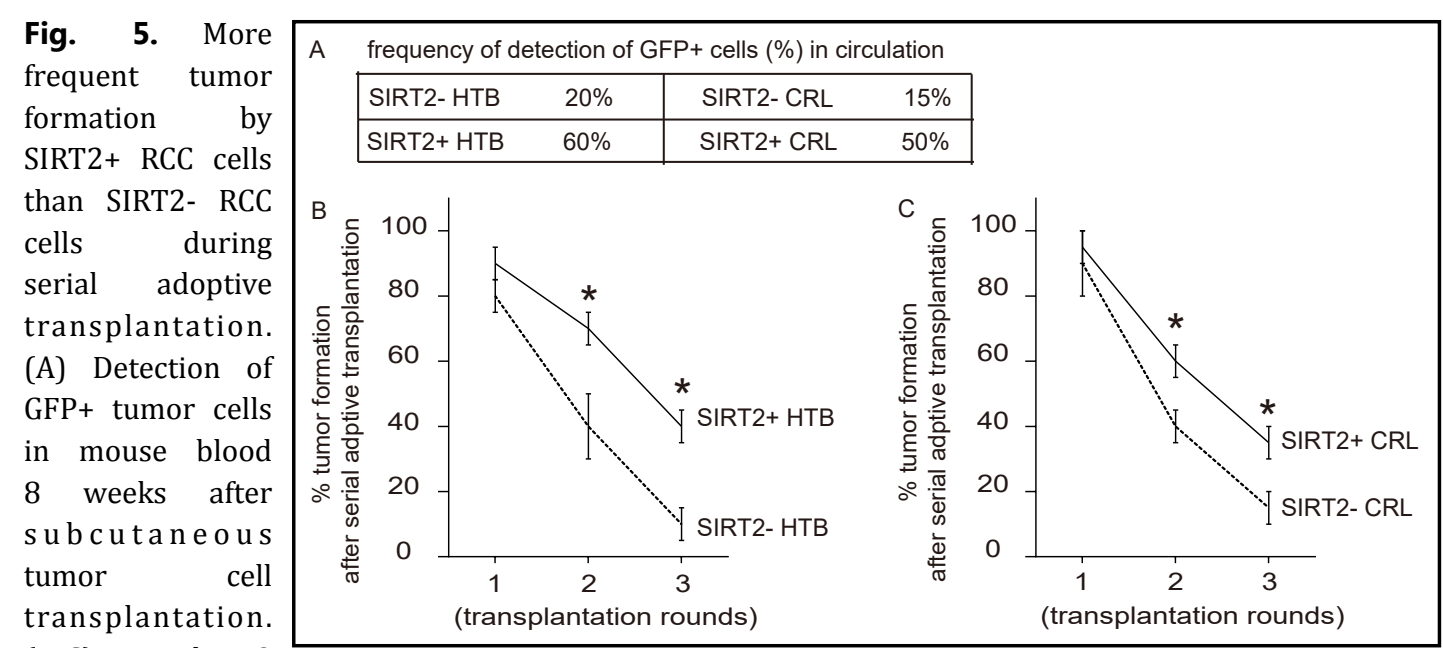

(B-C) In the 3

rounds of serial adoptive transplantation, the frequency of developing tumor by HTB cells (C) and by CRL cells (C). “*” indicated $\mathrm{p}<0.05 . \mathrm{N}=30$.

transplanted SIRT2- and SIRT2+ cells, and these cells were subcutaneously transplanted back to new nude mice. The mice were monitored for the formation of $2^{\text {nd }}$ generation of tumor for 8 weeks. Then, the $2^{\text {nd }}$ generation of tumor was removed and 20 tumor cells were purified for transplantation to form $3^{\text {rd }}$ generation of tumor. A total of 3 rounds of serial adoptive transplantation were performed and the tumor formation was monitored. Generally, more frequent tumor formation was observed in SIRT2+ cells during the serial adoptive transplantation, compared to that of in SIRT2- cells (Fig. 5B-C). All these results confirmed that the CSC cells could be purified with the marker of SIRT2 in RCC.

\section{Discussion}

Application of chemotherapeutic and biological agents is a critical therapeutic strategy for RCC. However, CSCs, which contribute to ineffective treatment, chemo-resistance, cancer metastasis and recurrence, were found to be difficult of removal or elimination by most of these strategies [25]. Consequently, the CSCs targeted approaches have great potential in improving the prognosis of RCC patients.

Previous studies have demonstrated CD133 and CD24 as CSC markers in RCC. However, lack of specificity of these markers only leads to enrichment of the CSC cells rather than purification of the CSC cells. Hence, additional CSC markers need to be identified to further enrich this CSC-like cells population, which is important for further improving the prognosis of cancer therapy.

In this study, we aimed to examine SIRT2 as a CSC marker in RCC. This hypothesis stemmed from recent studies that showing involvement of SIRT2 in tumorigenesis. The SIRT2 was found to stabilize Slug to increase EMT and aggressiveness of breast cancer [22]. In addition, SIRT2 was found to work with cortactin to promote prostate cancer progression [16]. Since the primary function of SIRT2 is to regulate cell cycle, we feel that SIRT2 may be essential for controlling tumor stemness.

Indeed, here, CSCs were characterized with several standard methods from both in vitro and in vivo, including the formation of tumor sphere and chemo-resistance (in vitro), tumor formation in serial adoptive transplantation (in vivo). The CSCs could be significantly enriched in the SIRT2+ cells. Moreover, SIRT2+ RCC cells were more frequently observed in the circulation of the mice. Our results indicated that circulating tumor stem-like cells could be purified in SIRT2+ RCC cells, instead of only CSCs. In this study, two common human RCC lines were included to increase the reliability of the conclusion. Our results from clinical 


\section{Cellular Physiology Cell Physiol Biochem 2018;49:2348-2357

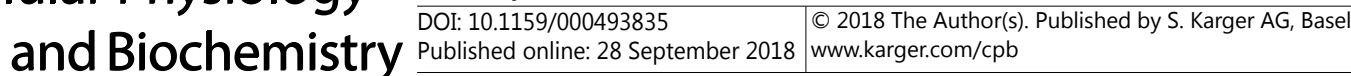

Wei et al.: SIRT2 as a CSC Marker in RCC

specimens further indicated an importance of using SIRT2 in the prognosis of the RCC and RCC stages. Future studies should be performed to analyze the downstream targets of SIRT2 in RCC, which is associated with tumor growth and invasiveness.

\section{Conclusion}

This study provided evidence that SIRT2+ could be a specific CSC marker in RCC. The outcome of current RCC therapy could be improved through selective elimination of SIRT2+ RCC cells.

\section{Disclosure Statement}

The authors have declared no conflict of interests.

\section{References}

1 Su D, Stamatakis L, Singer EA, Srinivasan R: Renal cell carcinoma: molecular biology and targeted therapy. Curr Opin Oncol 2014;26:321-327.

- Keefe SM, Nathanson KL, Rathmell WK: The molecular biology of renal cell carcinoma. Semin Oncol 2013;40:421-428.

3 Stewart GD, O’Mahony FC, Powles T, Riddick AC, Harrison DJ, Faratian D: What can molecular pathology contribute to the management of renal cell carcinoma? Nat Rev Urol 2011;8:255-265.

4 Pecqueux M, Fritzmann J, Adamu M, Thorlund K, Kahlert C, Reissfelder C, Weitz J, Rahbari NN: Free intraperitoneal tumor cells and outcome in gastric cancer patients: a systematic review and meta-analysis. Oncotarget 2015;6:35564-35578.

5 Zhou J, Ma X, Bi F, Liu M: Clinical significance of circulating tumor cells in gastric cancer patients. Oncotarget 2017;8:25713-25720.

-6 Rathmell WK, Malkowicz SB, Holroyde C, Luginbuhl W, Vaughn DJ: Phase II trial of 5-fluorouracil and leucovorin in combination with interferon-alpha and interleukin-2 for advanced renal cell cancer. Am J Clin Oncol 2004;27:109-112.

7 Wang T, Shigdar S, Gantier MP, Hou Y, Wang L, Li Y, Shamaileh HA, Yin W, Zhou SF, Zhao X, Duan W: Cancer stem cell targeted therapy: progress amid controversies. Oncotarget 2015;6:44191-44206.

-8 Saeednejad Zanjani L, Madjd Z, Abolhasani M, Shariftabrizi A, Rasti A, Asgari M: Expression of CD105 cancer stem cell marker in three subtypes of renal cell carcinoma. Cancer Biomark 2018;21:821-837.

-9 Ottaiano A: Finding markers for cancer stem cells in renal cell carcinoma: looking beyond CD133. Cell Cycle 2010;9:4431.

10 Galleggiante V, Rutigliano M, Sallustio F, Ribatti D, Ditonno P, Bettocchi C, Selvaggi FP, Lucarelli G, Battaglia M: CTR2 identifies a population of cancer cells with stem cell-like features in patients with clear cell renal cell carcinoma. J Urol 2014;192:1831-1841.

11 Donmez G, Outeiro TF: SIRT1 and SIRT2: emerging targets in neurodegeneration. EMBO Mol Med 2013;5:344-352.

$>12$ Harting K, Knoll B: SIRT2-mediated protein deacetylation: An emerging key regulator in brain physiology and pathology. Eur J Cell Biol 2010;89:262-269.

13 Inoue T, Hiratsuka M, Osaki M, Oshimura M: The molecular biology of mammalian SIRT proteins: SIRT2 in cell cycle regulation. Cell Cycle 2007;6:1011-1018.

14 Hoffmann G, Breitenbucher F, Schuler M, Ehrenhofer-Murray AE: A novel sirtuin 2 (SIRT2) inhibitor with p53-dependent pro-apoptotic activity in non-small cell lung cancer. J Biol Chem 2014;289:5208-5216.

15 McGlynn LM, Zino S, MacDonald AI, Curle J, Reilly JE, Mohammed ZM, McMillan DC, Mallon E, Payne AP, Edwards J, Shiels PG: SIRT2: tumour suppressor or tumour promoter in operable breast cancer? Eur J Cancer 2014;50:290-301. 


\section{Cellular Physiology Cell Physiol Biochem 2018;49:2348-2357

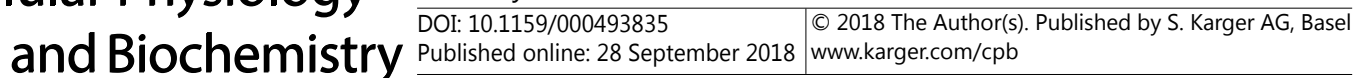

Wei et al.: SIRT2 as a CSC Marker in RCC

16 Hou H, Chen W, Zhao L, Zuo Q, Zhang G, Zhang X, Wang H, Gong H, Li X, Wang M, Wang Y, Li X: Cortactin is associated with tumour progression and poor prognosis in prostate cancer and SIRT2 other than HADC6 may work as facilitator in situ. J Clin Pathol 2012;65:1088-1096.

17 Zuo Q, Wu W, Li X, Zhao L, Chen W: HDAC6 and SIRT2 promote bladder cancer cell migration and invasion by targeting cortactin. Oncol Rep 2012;27:819-824.

18 Li Y, Zhang M, Dorfman RG, Pan Y, Tang D, Xu L, Zhao Z, Zhou Q, Zhou L, Wang Y, Yin Y, Shen S, Kong B, Friess H, Zhao S, Wang L, Zou X: SIRT2 Promotes the Migration and Invasion of Gastric Cancer through RAS/ERK/ JNK/MMP-9 Pathway by Increasing PEPCK1-Related Metabolism. Neoplasia 2018;20:745-756.

19 Liu CW, Lin YC, Hung CM, Liu BL, Kuo SC, Ho CT, Way TD, Hung CH: CHM-1, a novel microtubuledestabilizing agent exhibits antitumor activity via inducing the expression of SIRT2 in human breast cancer cells. Chem Biol Interact 2018;289:98-108.

20 Damodaran S, Damaschke N, Gawdzik J, Yang B, Shi C, Allen GO, Huang W, Denu J, Jarrard D: Dysregulation of Sirtuin 2 (SIRT2) and histone H3K18 acetylation pathways associates with adverse prostate cancer outcomes. BMC Cancer 2017;17:874.

-21 Zhang LL, Zhan L, Jin YD, Min ZL, Wei C, Wang Q, Chen YJ, Wu QM, Hu XM, Yuan Q: SIRT2 mediated antitumor effects of shikonin on metastatic colorectal cancer. Eur J Pharmacol 2017;797:1-8.

22 Zhou W, Ni TK, Wronski A, Glass B, Skibinski A, Beck A, Kuperwasser C: The SIRT2 Deacetylase Stabilizes Slug to Control Malignancy of Basal-like Breast Cancer. Cell Rep 2016;17:1302-1317.

23 Humeniuk R, Mishra PJ, Bertino JR, Banerjee D: Epigenetic reversal of acquired resistance to 5-fluorouracil treatment. Mol Cancer Ther 2009;8:1045-1054.

24 Srimuangwong K, Tocharus C, Yoysungnoen Chintana P, Suksamrarn A, Tocharus J: Hexahydrocurcumin enhances inhibitory effect of 5-fluorouracil on HT-29 human colon cancer cells. World J Gastroenterol 2012;18:2383-2389.

25 Saikawa Y, Fukuda K, Takahashi T, Nakamura R, Takeuchi H, Kitagawa Y: Gastric carcinogenesis and the cancer stem cell hypothesis. Gastric Cancer 2010;13:11-24. 